\title{
DNA methylation signature is prognostic of choroid plexus tumor aggressiveness
}

\author{
Malgorzata Pienkowska', Sanaa Choufani ${ }^{1}$, Andrei L. Turinsky ${ }^{1,2}$, Tanya Guha ${ }^{1}$, Diana M. Merino ${ }^{3}$, Ana Novokmet ${ }^{1 \wedge}$, \\ Michael Brudno ${ }^{1,2,4}$, Rosanna Weksberg ${ }^{1,5,8}$, Adam Shlien ${ }^{1,6}$, Cynthia Hawkins ${ }^{1,6}$, Eric Bouffet ${ }^{7,8}$, Uri Tabori 1,7,8, \\ Richard J. Gilbertson ${ }^{10}$, Jonathan L. Finlay ${ }^{11}$, Nada Jabado ${ }^{12}$, Christian Thomas ${ }^{13}$, Martin Sill ${ }^{14,15}$, David Capper ${ }^{16,17}$, \\ Martin Hasselblatt ${ }^{13}$ and David Malkin ${ }^{1,7,8,9^{*}}$ (D)
}

\begin{abstract}
Background: Histological grading of choroid plexus tumors (CPTs) remains the best prognostic tool to distinguish between aggressive choroid plexus carcinoma (CPC) and the more benign choroid plexus papilloma (CPP) or atypical choroid plexus papilloma (aCPP); however, these distinctions can be challenging. Standard treatment of CPC is very aggressive and often leads to severe damage to the young child's brain. Therefore, it is crucial to distinguish between CPC and less aggressive entities (CPP or aCPP) to avoid unnecessary exposure of the young patient to neurotoxic therapy. To better stratify CPTs, we utilized DNA methylation (DNAm) to identify prognostic epigenetic biomarkers for CPCS.
\end{abstract}

Methods: We obtained DNA methylation profiles of 34 CPTs using the HumanMethylation450 BeadChip from Illumina, and the data was analyzed using the Illumina Genome Studio analysis software. Validation of differentially methylated CpG sites chosen as biomarkers was performed using pyrosequencing analysis on additional 22 CPTs. Sensitivity testing of the CPC DNAm signature was performed on a replication cohort of 61 CPT tumors obtained from Neuropathology, University Hospital Münster, Germany.

Results: Generated genome-wide DNAm profiles of CPTs showed significant differences in DNAm between CPCs and the CPPs or aCPPs. The prediction of clinical outcome could be improved by combining the DNAm profile with the mutational status of TP53. CPCs with homozygous TP53 mutations clustered as a group separate from those carrying a heterozygous TP53 mutation or CPCS with wild type TP53 (TP53-wt) and showed the worst survival outcome. Specific DNAm signatures for CPCs revealed AK1, PER2, and PLSCR4 as potential biomarkers for CPC that can be used to improve molecular stratification for diagnosis and treatment.

Conclusions: We demonstrate that combining specific DNAm signature for CPCs with histological approaches better differentiate aggressive tumors from those that are not life threatening. These findings have important implications for future prognostic risk prediction in clinical disease management.

Keywords: DNA methylation, Choroid plexus tumors, HumanMethylation450 arrays, Quantitative sodium bisulfite pyrosequencing

\footnotetext{
* Correspondence: david.malkin@sickkids.ca

${ }^{1}$ Genetics and Genome Biology Program, Hospital for Sick Children, PGCRL,

686 Bay Street, Toronto, Ontario M5G 0A4, Canada

${ }^{7}$ Division of Hematology/Oncology, Hospital for Sick Children, 555 University

Avenue, Toronto, Ontario M5G 1X8, Canada

Full list of author information is available at the end of the article
}

(c) The Author(s). 2019, corrected publication 2019. Open Access This article is distributed under the terms of the Creative Commons Attribution 4.0 International License (http://creativecommons.org/licenses/by/4.0/), which permits unrestricted use, distribution, and reproduction in any medium, provided you give appropriate credit to the original author(s) and the source, provide a link to the Creative Commons license, and indicate if changes were made. The Creative Commons Public Domain Dedication waiver (http://creativecommons.org/publicdomain/zero/1.0/) applies to the data made available in this article, unless otherwise stated. 


\section{Introduction}

Choroid plexus tumors (CPTs) are rare neoplasms of the central nervous system. Within this family of tumors, choroid plexus carcinoma (CPC) is a malignant neoplasm, categorized as a grade III tumor by the World Health Organization (WHO). In contrast, choroid plexus papilloma (CPP) is benign and classified as a grade I tumor, while atypical choroid plexus papilloma $(\mathrm{aCPP})$ is a grade II tumor [1]. CPTs account for $0.4-0.6 \%$ of all brain tumors; however, in children, CPTs represent 1 to $4 \%$ of all childhood brain tumors, with up to $20 \%$ occurring during the first year of life [1]. CPCs account for $20-40 \%$ of all choroid plexus tumors in children [1].

Distinction between these tumor subtypes for accurate diagnosis is essential but can be challenging. Current diagnosis relies on assessment of histopathological features, i.e., mitotic activity, cellularity, and nuclear pleomorphism as well as the presence of necrosis and blurring of the papillary growth pattern [2]. Although overall long-term survival for CPPs is relatively favorable $(85-100 \%)$ after surgical resection alone, CPCs are significantly more aggressive, with a greater tendency for recurrence and less than $50 \%$ of patients survive even in the context of combined surgery, chemo- and radiation therapy [3-6]. Most of these children are younger than 3 years of age, and the long-term damaging effects of this therapy on growth and the developing brain are of immense concern, highlighting the need for better biologic risk stratification for tumors in these young patients.

Over $50 \%$ of CPC tumors carry somatic mutations in the TP53 tumor suppressor gene, and TP53 mutant CPCs have been associated with increased genome instability and poor prognosis [7]. Germline TP53 mutations have also been observed in $\sim 50 \%$ of children with CPC which is now considered a component tumor of Li-Fraumeni syndrome (LFS) [8]. We have previously reported that CPTs are highly unstable and harbor unique patterns of chromosome-wide gains and losses [9]. In fact, we demonstrated that differences in copy number $(\mathrm{CN})$ and gene expression distinguish CPCs from CPPs and aCPPs. Nevertheless, despite the use of similar treatment protocols for all patients with $\mathrm{CPC}$, clinical outcomes vary, and our previous findings demonstrate that the clinical variability may be driven by molecular heterogeneity of CPCs.

To improve outcome prediction, more accurate molecular distinction among CPTs is needed. The power of DNAm to identify novel, more molecularly defined tumor subtypes has been established and led to improved stratification and specific tailoring of therapy for patients with a wide range of cancers [10-12]. Recently, Thomas et al. identified three clinically distinct subgroups of choroid plexus tumors by array-based DNAm profiling; however, the methylation groups did not entirely recapitulate the three different histologically defined WHO entities [13].
Based on these observations and our previously reported work, we performed a detailed analysis of the genome-wide methylation profile in our cohort of CPTs with the goal of determining a more accurate classification of the CPT subtypes.

In this study, we performed a comprehensive analysis of DNAm in CPTs and discovered a highly sensitive and specific DNAm signature for CPCs which is able to segregate CPC from other CPT tumors as well as other brain tumors. This signature includes AK1, PER2, and PLSCR4 as prospective diagnostic biomarkers for CPC and potentially tractable therapeutic targets.

\section{Methods}

\section{Patients, tissues, and sample preparation}

Institutional Research Ethics Board approval was obtained for the study. Clinical data and tumor samples were collected from many sources, including two large pediatric neuro-oncology centers: the Hospital for Sick Children (SickKids), Toronto, Ontario, and the Children's Hospital of Los Angeles (CHLA), Los Angeles, CA. The other contributing centers were as follows: St. Jude Children's Research Hospital, Memphis, TN; the Collaborative Human Tissue Network (CHTN) in Columbus, OH; Schneider Children's Medical Center, Tel-Aviv, Israel; Montreal Children's Hospital, Montreal, Quebec; and University of Colorado Health Sciences Center, Denver, CO. Informed consent was obtained from the parents/legal guardians of all patients. Pathologic review of CPTs was conducted by Dr. C. Hawkins. In all other institutions, expert neuropathologists critically examined each case. All samples were processed as described in detail in our previous work [9]. DNA was isolated from either fresh snap frozen $(n=53)$ or formalin-fixed paraffin-embedded (FFPE; $n=2$ ) or optimal cutting temperature (OCT) compound $(n=1)$ tumor samples. Tumor DNA was extracted using standard phenol-chloroform extraction from fresh frozen samples and the RecoverAll Total Nucleic Acid Isolation Kit for FFPE (Ambion) from FFPE samples.

Our primary cohort comprised 34 samples, including 15 CPPs, 5 aCPPs, and 14 CPCs. An additional validation cohort comprised 22 samples, including 4 CPPs, 3aCPPs, and 15 CPCs. We also used a replication cohort of 61 CPTs from Neuropathology, University Hospital Münster, Germany, for sensitivity testing of the CPC-specific DNAm signature [13]. Patient characteristics are provided in Additional file 1: Tables $\mathrm{S} 1$ and $\mathrm{S} 2$.

\section{TP53 gene sequencing}

Sequencing of the coding region of TP53 (exons 2-11 as well as up to 50 bases into spanning introns of the TP53 gene) was performed in the molecular diagnostic laboratory at The Hospital for Sick Children in Toronto by 
direct Sanger sequencing of genomic DNA as previously described [7].

\section{DNA methylation}

Genomic DNA $(\sim 1 \mu \mathrm{g})$ from CPT patients (Additional file 1: Table S1) was treated using sodium bisulfite (Qiagen) converting unmethylated cytosine to uracil but leaving methylated cytosine intact. These samples were then hybridized to the HumanMethylation450 BeadChip from Illumina at the Centre for Applied Genomics (TCAG) at the Hospital for Sick Children using the manufacturer's recommended protocol. Genome-wide DNAm profiles for all 34 primary samples are available through Gene Expression Omnibus (GEO: http://www.ncbi.nlm.nih.gov/geo/), accession number GSE61044.

\section{Genome-wide DNA methylation analysis}

The data generated from the HumanMethylation450 BeadChip arrays were analyzed using the Illumina Genome Studio analysis software and included normalization, quality control measurements, and background correction. We removed probes targeting the $\mathrm{X}$ or $\mathrm{Y}$ chromosomes, probes near polymorphic sites (targeting CpGs within $5 \mathrm{pb}$ of SNPs that have $\geq 1 \%$ minor allele frequency in 1000 Genome Project), internal control probes, and non-specific cross-reactive probes [14]. This filtering process resulted in the retention of $414319 \mathrm{CpG}$ methylation sites for further analysis. For each CpG site, the DNAm level was expressed as the average percentage of methylated cytosines, known as the beta value $(\beta=\mathrm{mC} /(\mathrm{mC}+\mathrm{C}))$. Mapping of $\mathrm{CpG}$ sites to regulatory genomic regions was done using the Illumina annotation.

To determine the sites that are differentially methylated between the carcinoma and papilloma groups, we used a non-parametric Mann-Whitney $U$ test at each CpG probe. For all subsequent investigations except the biomarker discovery, we applied the statistical significance level at a $p$ value $<0.05$ (after FDR correction) and an additional cutoff of at least $30 \%$ average methylation difference $(\Delta \beta)$ between the groups.

For biomarker discovery, more stringent criteria were used, with a $p$ value $<0.001$ and average methylation difference $\Delta \beta \geq 40 \%$. Data were visualized in Qlucore Omics Explorer 3.3 and Partek Genomics Suite 6.6 software using heatmaps and principal component analysis (PCA) plots.

All differentially methylated genes identified in this study were analyzed using Ingenuity Pathway Analysis (IPA) software to identify pathways that are involved in the etiology of the carcinomas.

\section{Predictive modeling of the CPC-specific signature}

Predictive analysis was performed using the Weka machine learning suite (www.cs.waikato.ac.nz/ml/weka) and
$\mathrm{R}$ package caret [15]. Predictive models were built using CpGs as data attributes. To avoid overfitting, we used options CfsSubsetEval-BestFirst to search for the most predictive non-redundant subset of $\mathrm{CpGs}$ using the correlation-based feature selection method. For the purposes of predictive analysis, all $34 \mathrm{CPT}$ data samples were labeled as either "carcinoma" (CPC) or "papilloma" (including CPP and aCPP). Leave-one-out (LOO) crossvalidation was applied where one sample was withheld; a new epigenetic signature was identified, and a predictive model was built on the remaining 33 samples and then applied to the withheld sample to predict its status as "carcinoma" or "papilloma." Repeating this process for each of the 34 CPTs gave the measure of the predictive accuracy.

\section{Multivariate factor analysis}

Using 95 CPT samples of the combined datasets of the 34-sample discovery cohort and the 61-sample replication cohort (35 CPC, 33 CPP, 27 aCPP), we performed multivariate factor analysis of the DNAm levels along with other sample genotype and phenotype attributes to identify possible correspondence between them. Beta values in each of the 59 signature CpGs were analyzed jointly with attributes such as age, TP53 mutation status, recurrence event status, and death event status (Additional file 1: Table S3). See Additional file 3 for details.

\section{Validation of differentially methylated CpG sites chosen as biomarkers (pyrosequencing analysis)}

Three genes were chosen as biomarkers that demonstrate the greatest degree of segregation between CPCs and CPPs, and the corresponding CpG sites were validated for differential methylation between these tumor groups using both the initial discovery panel of 34 samples and a validation set of 22 samples. Quantitative sodium bisulfite pyrosequencing was performed for AK1 (cg14578146), PER2 (cg11903188), and PLSCR4 (cg07038342). All targeted assays were designed using the PyroMark Assay Design Software 1.0 (Qiagen). All primer sets are listed in Additional file 1: Table S4. Sodium bisulfite-modified genomic DNA was amplified using Hot-Start Taq Master Mix (Qiagen) as previously described [16]. Regions of interest were amplified by PCR, and pyrosequencing was carried out using the PyroMark Q24 pyrosequencer (Qiagen) according to the manufacturer's protocol (Pyro-Gold reagents). Output data were analyzed using PyroMark Q24 1.0.10 Software (Qiagen), which calculates the methylation percentage $\beta$ for each $\mathrm{CpG}$ site, allowing quantitative comparisons.

\section{Survival analysis}

Survival analysis was performed using the Kaplan-Meier method, and curves were compared using both log-rank 
and Wilcoxon-Gehan chi-square tests. Overall survival (OS) measured time from initial diagnosis to death from any cause or last follow-up.

\section{Results}

Identification of methylation signature for CPCs and TP53 mutation groups in CPCs

Analysis of the genome-wide DNAm of primary CPTs did not show significant differences between CPPs and aCPPs (Mann-Whitney $p$ value $\geq 0.55$ after FDR correction in all 485577 CpG probes) but there was a significant difference in the $\mathrm{CpG}$ methylation profile between CPCs and CPPs or aCPPs (Mann-Whitney $p$ value $\leq 0.048$, FDR corrected in 51479 CpG sites). Although there were no significant differences in genome-wide DNAm between CPPs and aCPPs, there was a CPC-specific signature in comparison to CPP.

A volcano plot of the genome-wide DNAm profile revealed a general shift towards higher DNAm levels (hypermethylation) in the CPCs as compared to the CPPs (Additional file 2: Figure S1).

Using an average beta value difference of 0.3 or greater and an FDR adjusted $p$ value $<0.05$, we identified a total of $3361 \mathrm{CpG}$ probes that showed significant differences in methylation between CPCs and CPPs. Among these 3361 CpGs, 1388 (or 41\%) were hypomethylated and the remaining 1973 CpG sites (or 59\%) were hypermethylated in CPCs compared to CPPs. Two-way clustering performed using Pearson's correlation and average linkage for both the sample tree and the gene tree revealed segregation between the majority $(86 \%)$ of CPCs and CPPs or aCPPs (Fig. 1).

Since over $50 \%$ of CPC tumors carry somatic TP53 mutations, and TP53 mutant CPCs have been associated with poor prognosis [7], we sought to determine whether the clustering observed within the CPC group correlated with TP53 mutation status.

As shown in Fig. 1a, b, CPCs harboring homozygous mutations in TP53 clustered as a group separate from those carrying a heterozygous TP53 mutation or CPCs with TP53-wt. Survival analysis performed on CPTs (Fig. 2) showed significantly worse outcomes for methylation group 1 and 2, which contained only CPC samples (with mutated TP53 or TP53-wt), than for the methylation group 3 that contained predominantly CPPs or aCPPs and had no death events. Methylation group 1 comprising only CPCs with homozygous TP53 mutation had a particularly poor prognosis compared to methylation group 2 which contained CPCs with heterozygous TP53 mutation or the TP53-wt (log-rank $\chi^{2}=16.7$ with $\mathrm{df}=2, p$ value 0.00023 ; and Wilcoxon-Gehan $\chi^{2}=15.5$ with $\mathrm{df}=2, p$ value 0.00043 ).
Pathway and biological function analysis of differentially methylated genes

To investigate whether genes affected by alterations of DNAm in CPCs tend to fall within specific molecular pathways, we next performed pathway analysis using Ingenuity Pathway Analysis (IPA) software on a 1328 gene set overlapping the $3361 \mathrm{CpG}$ probes. We identified nine canonical pathways that were significantly enriched in CPCs (FDR corrected $p$ value $<0.05$, with between 14 and 29 differentially methylated genes in each pathway) in comparison to CPPs (Fig. 3a). As shown in Fig. 3a, the most significantly affected pathway was GABA receptor signaling where 14 genes showed differential methylation between CPCs and CPPs or aCPPs. We observed hypomethylation in the body of $G A B A(A)$ receptor subunits alpha 4-5 and gamma 3, as well as hypermethylation in the promoter region of subunit gamma 2 in CPCs compared to CPPs (Additional file 1: Table S5).

IPA Biological Function analysis revealed 54 functional categories that were significantly altered (FDR corrected $p$ value $<0.05)$ in CPCs. The most significantly enriched categories in CPCs (with $p$ values below 0.001 and including between 94 and 408 differentially methylated genes) were associated with cellular development, cellular growth and proliferation, cancer, cell death and survival, and cellular assembly and organization (Fig. 3b).

\section{Genomic enrichment of the CPC DNA methylation signature}

In order to identify regulatory regions that are enriched in the CPC DNAm signature, we used the Illumina annotation for regulatory regions and compared the 3361 sites with significant differential DNAm in CPCs against the full Illumina $450 \mathrm{~K}$ array dataset. We identified significant enrichment of $\mathrm{CpG}$ sites associated with CPCs in enhancer regions, DNase I hypersensitive sites (DHS) (which are associated with open chromatin and hence active transcription), cancer-specific differentially methylated regions (cDMR), reprogrammed-specific differentially methylated regions (rDMR), and non-CpG island sites. However, we did not find any enrichment at $\mathrm{CpG}$ island shores and shelves (Additional file 2: Figure S2).

\section{Correlation between DNA methylation and gene expression}

To assess the impact of differential methylation on gene expression, we examined the $3361 \mathrm{CpG}$ sites in a dataset of $40 \mathrm{CPTs}$ analyzed previously using Affymetrix Exon1 microarrays [9]. We identified 26 samples from this study that had expression profiling data available; of those, we had 11 CPCs, 12 CPPs, and 4 aCPPs. Differential expression between CPCs and CPPs was determined at the significance level with FDR corrected $p$ value $<0.05$. We identified 57 single genes showing correlation between 


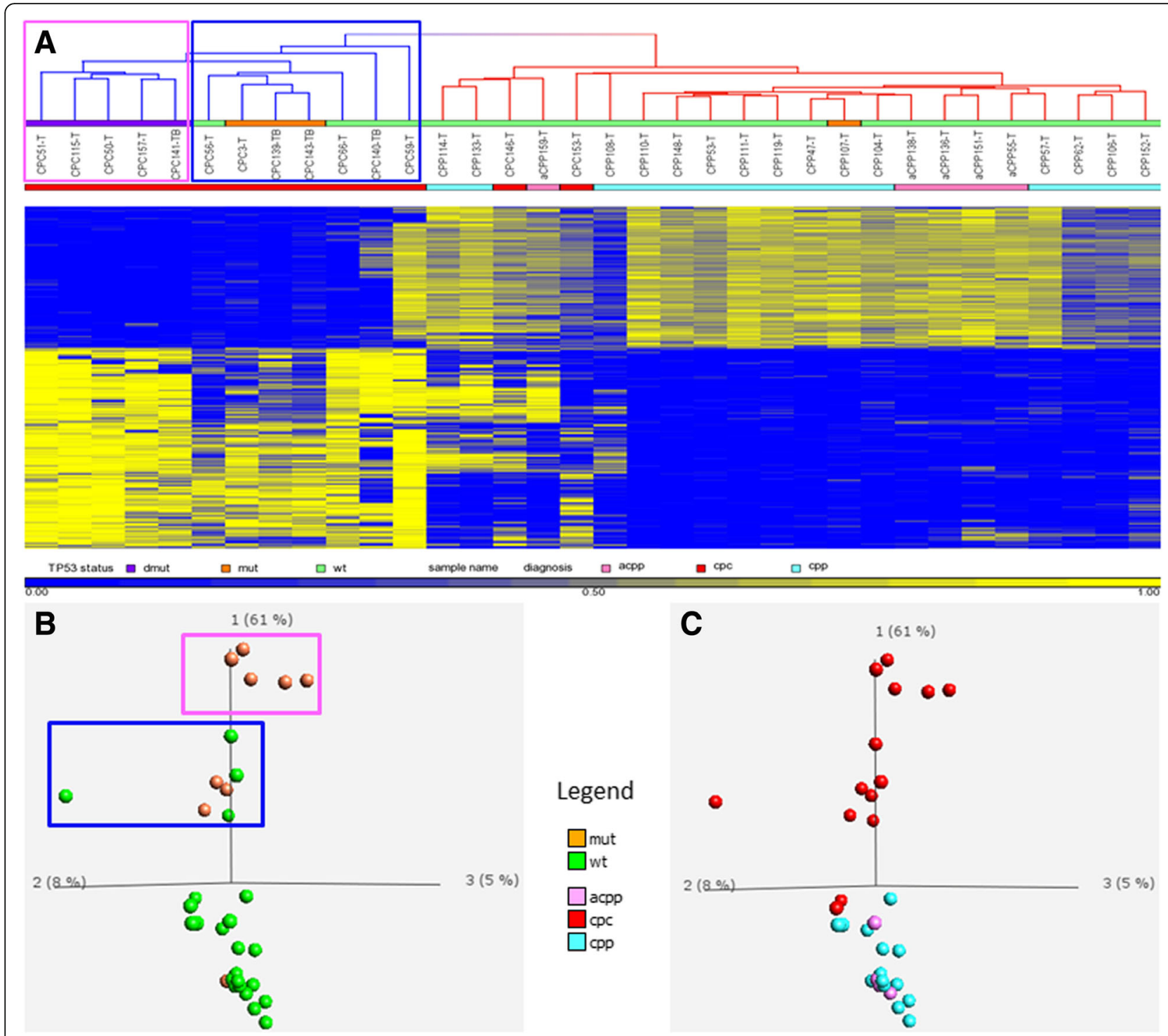

Fig. 1 Two-way clustering performed on 34 CPT samples using Pearson's correlation and average linkage (a) and PCA (b, c) using the top 3361 most differentially methylated CpGs ( $p<0.05$ after FDR correction and at least 30\% methylation difference) shows segregation between majority of carcinomas (срс) and papillomas (cpp and acpp). In addition, we observed segregation within the CPC group based on TP53 status. Homozygous TP53-mut (mutant) = violet bar (a) and outlined in pink box $(\mathbf{a}, \mathbf{b})$; heterozygous TP53-mut = orange bar $(\mathbf{a})$ and outlined in blue box $(\mathbf{a}, \mathbf{b})$; TP53-wt ( wild type) $=$ green bar $(\mathbf{a})$ and green dots $(\mathbf{b})$; diagnosis: $\operatorname{acpp}=\operatorname{pink}, \mathrm{cpc}=$ red, and $\mathrm{cpp}=$ turquoise $(\mathbf{a}-\mathbf{c})$. The numbers 1, 2, and 3 in PCA plots represent component 1, component 2, and component 3

methylation and expression levels in CPCs, suggesting an epigenetic regulation of gene expression for these important genes in CPCs. Namely, 32 genes displayed differential methylation in their promoter region (comprising the region $1500 \mathrm{bp}$ upstream of the transcription start site (TSS), the 5'UTR region, and the first exon), which was inversely correlated with their expression in CPCs (Fig. 4a and Additional file 1: Table S6). Meanwhile, 25 genes demonstrated differential methylation of probes within the gene body, which was positively correlated with their expression (Fig. 4b and Additional file 1: Table S7).

\section{CPC-specific DNA methylation signature}

We further increased the differential methylation stringency ( $p$ value $<0.001$ after FDR correction and $\Delta \beta>0.4$ by magnitude), which allowed us to extract 59 high-confidence differentially methylated CpG sites encompassing 33 candidate genes (Fig. 5 and Additional file 1: Table S8).

We chose CpG sites representing 3 genes $(A K 1-$ adenylate kinase, PER2-period circadian clock 2, and PLSCR4-phospholipid scramblase 4) for the validation of their differentially methylated status by targeted 

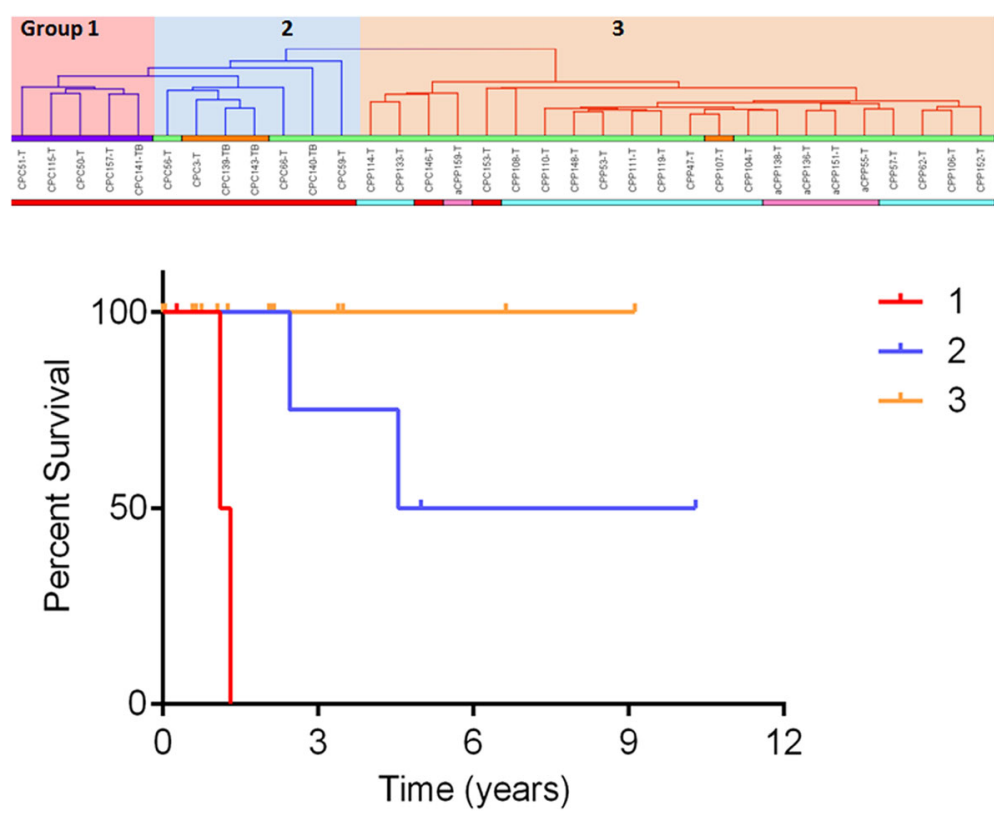

Kaplan-Meier (KM) curve depicting overall survival (OS) estimates of patients with CPT the three KM curves were obtained with the log-rank chi-square $=16.7(\mathrm{df}=2), p$ value 0.0002; and Wilcoxon-Gehan chi-square $=15.5(\mathrm{df}=2), p$ value 0.00043. Group 1 (pink highlight in top panel, red line in KM plot $(n=5)$ ), CPCs with homozygous TP53-mut (mutant) = violet bar; group 2 (blue highlight in top panel, blue line in KM plot $(n=7)$ ), CPCs with heterozygous TP53-mut= orange bar and TP53-wt (wild type)=green bar; group 3 (orange highlight in top panel, orange line in KM plot $(n=22))$, CPPs-heterozygous TP53-mut = orange bar and TP53-wt = green bar and two CPCs with TP53-wt = green bar; diagnosis: $c p c=$ red, acpp $=$ pink, $c p p=$ turquoise

quantitative pyrosequencing. The selection was based on satisfying the following criteria: (1) CpG sites methylated in the promoter region, (2) inverse correlation between methylation and expression, and (3) known biological association with cancer. Results obtained by pyrosequencing using the discovery subset of 34 CPT tumors as well as the validation subset of $22 \mathrm{CPT}$ samples are presented in Additional file 2: Figure S3. For all three genes, bisulfite pyrosequencing validated the findings from the methylation array analysis and confirmed that the methylation at the tested CpG sites was higher in CPCs than in CPPs. In addition, we visualized DNAm profile of the 3 biomarker CpGs, obtained from the initial discovery subset of 34 CPTs using the HumanMethylation450 array and from the validation subset of 22 CPTs using pyrosequencing. We found that these 3 biomarkers were sufficient to segregate CPCs from the CPPs or aCPPs, proving them to be a highly specific minimal CPC DNAm signature (Additional file 2: Figure S4).

Direct comparison of the BeadChip and pyrosequencing values for the 34 cases for which data were available from both methods revealed a high correlation between the two methodologies, with $r^{2}$ between 0.90 and 0.99. Correlation plots for each tested CpG are shown in Additional file 2: Figure S5.

\section{Accuracy measurement of CPC DNA methylation signature}

We applied several types of machine learning models to the DNAm profiles of $14 \mathrm{CPC}$ samples and $20 \mathrm{CPP}$ or aCPP samples. Our goal was to predict the CPT status of a sample using the methylation values in signature CpGs as data attributes. Leave-one-out (LOO) cross-validation was used to determine the predictive accuracy.

First, we used only three CpGs as data attributes representing the minimal signature genes: $A K 1$ (cg14578146), PER2 (cg11903188), and PLSCR4 (cg07038342). Even with this minimal data representation, several predictive model types, such as logistic regression, multilayer perceptron neural network, and Naïve Bayes model, were able to predict carcinoma status on all left-out samples with perfect accuracy (area under $\mathrm{ROC}=100 \%$ in each case). The logistic regression model showed that cg11903188 (gene PER2) was the most predictive of the three, with an odds ratio $=7.33$, followed by cg07038342 (PLSCR4, odds ratio $=3.03)$ and $\operatorname{cg} 14578146(A K 1$, odds ratio $=1.20)$.

Next, we explored the predictive accuracy and robustness of the full epigenetic signature using LOO crossvalidation. A full new epigenetic signature was identified for each of the 34 cross-validation folds using the same parameters ( $p$ value $<0.001$ after FDR correction and $\Delta \beta>0.4$ by magnitude). This signature was then used to 

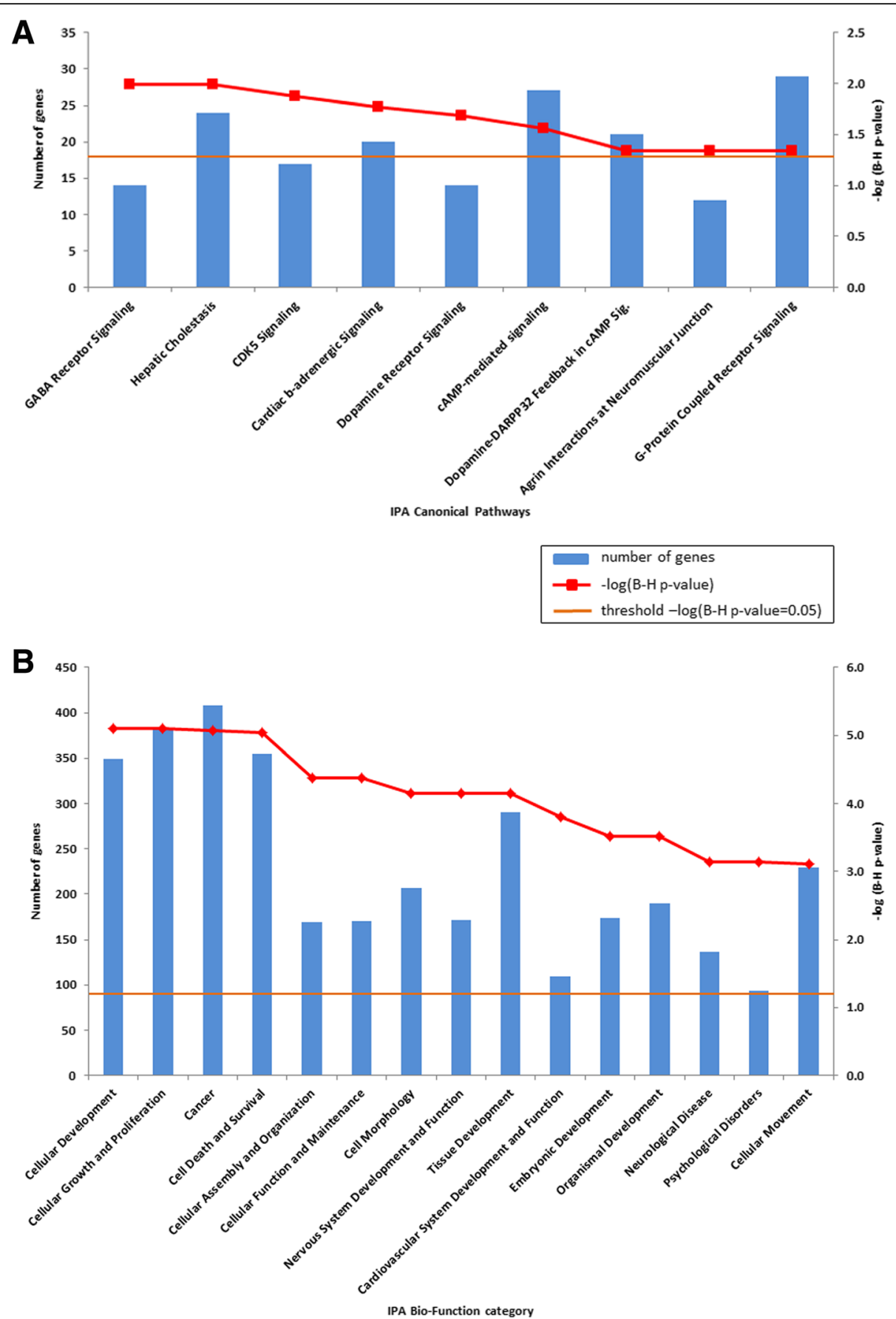

Fig. 3 Bar chart shows the enriched canonical pathways (a) or enriched biological function categories (b) in CPCs using Ingenuity Pathway Analysis (IPA). Major $Y$-axis on the left shows the number of differentially methylated genes. Secondary $Y$-axis on the right shows the significance levels (-log (B-H $p$ value 0.05$)$ ) of the canonical pathway (a) and (- $\log (\mathrm{B}-\mathrm{H} p$ value 0.001$))$ of the biological function category (b). The orange line shows the significance threshold cutoff of $-\log (\mathrm{B}-\mathrm{H} p$ value 0.05$)$. B-H, Benjamini-Hochberg multiple testing correction

build predictive models of several types, both without feature selection (i.e., using all CpGs as data attributes) and with feature selection to optimize model accuracy by using only the most predictive non-redundant CpGs. We applied several types of machine learning models available through the $\mathrm{R}$ package caret, such as logistic regression, support vector machines, and random forests, to detect carcinoma cases. In all such results, we achieved perfect $100 \%$ specificity, i.e., no false positives. However, the carcinoma sample CPC146- $\mathrm{T}$ was persistently misclassified by all models and occasionally the models also failed to detect another sample CPC153-T, 


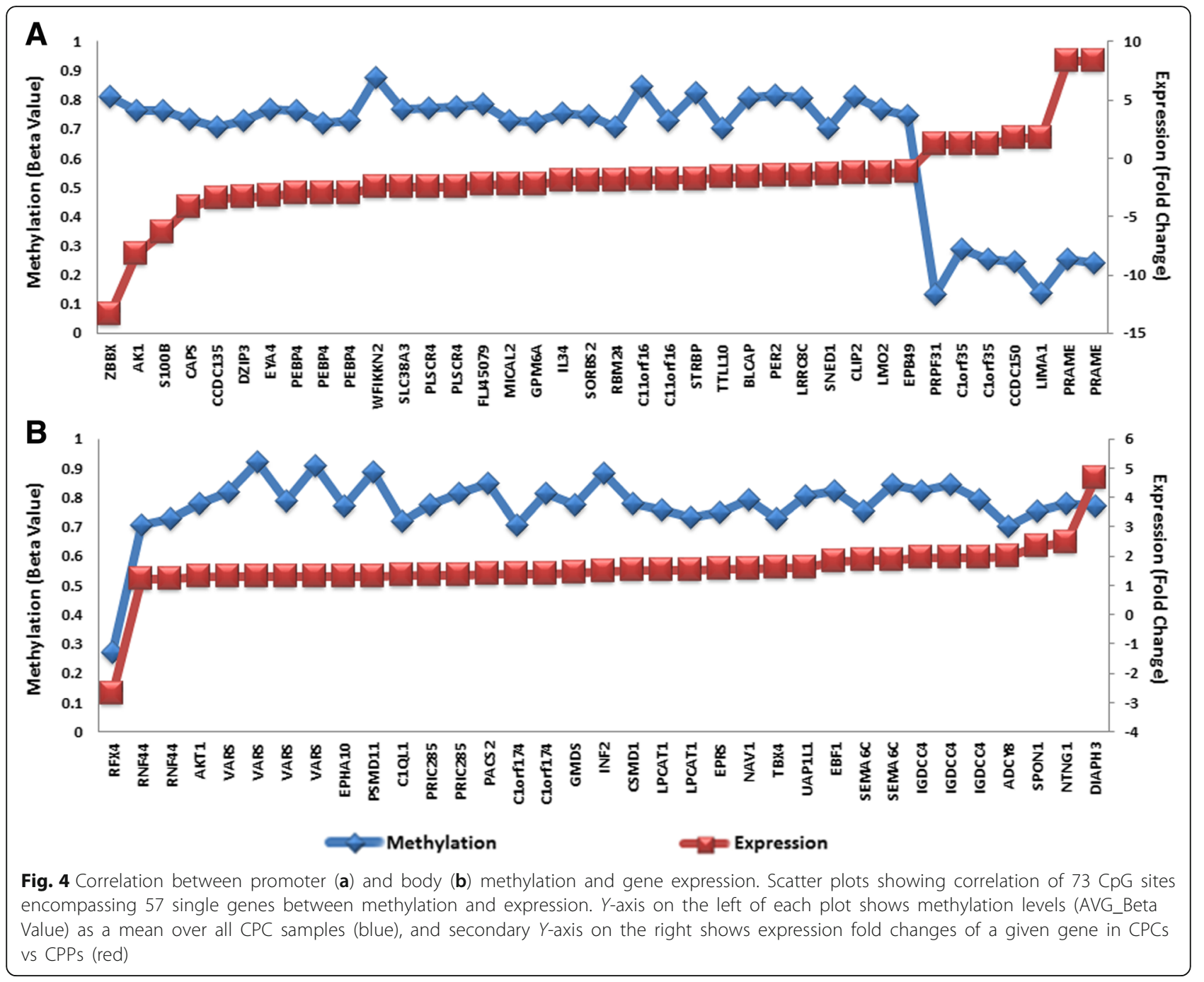

thereby giving a sensitivity estimate between 85.7 and 92.9\% ( 1 or 2 false positives out of 14 CPC samples). These observations match well with the results shown in Fig. 1 where the same two CPC samples are clustered within the papilloma group. Overall, our estimates of a perfect specificity and a high sensitivity suggest a good potential of the CPC-derived DNAm signature in predicting carcinomas.

Testing the specificity of CPC DNA methylation signature using external datasets

To further validate the specificity of the CPC biomarker signature, we extracted publicly available pediatric brain tumor DNAm datasets from the GEO database as well as from the TCGA (The Cancer Genome Atlas) Data Portal (http://portal.gdc.cancer.gov/) of adult origin and analyzed them jointly with our CPT methylation data. The brain tumor DNAm datasets derived from the Illumina HumanMethylation450 array consisted of 28 diffuse intrinsic pontine gliomas (DIPG) (GSE50022) and
67 pilocytic astrocytomas (GSE44684), 12 embryonal tumors with multilayered rosettes (ETMR) and 28 primitive neuroectodermal tumors (PNET) (GSE52556) from GEO, and 24 glioma and 30 low grade glioma (LGG) from TCGA. The extracted data were combined with our CPT data, all samples were restricted to the CpGs comprising the CPC-specific signature, and hierarchical clustering was used to examine the separation of CPTs from other brain tumors (Fig. 6). The heatmap of hierarchical clustering shows distinct DNAm patterns for CPCs. In addition, CPCs segregate from the majority of the other brain tumors, further confirming the high specificity of the CPC DNAm signature (Fig. 6).

Testing the sensitivity of the CPC DNA methylation signature using a replication cohort of CPT tumors We applied the CPC-specific DNAm signature to a cohort of CPTs derived from a recently published study [13]. This cohort consisted of $21 \mathrm{CPCs}, 22 \mathrm{aCPPs}$, and 18CPPs. Using this replication dataset, CPCs clustered together and 


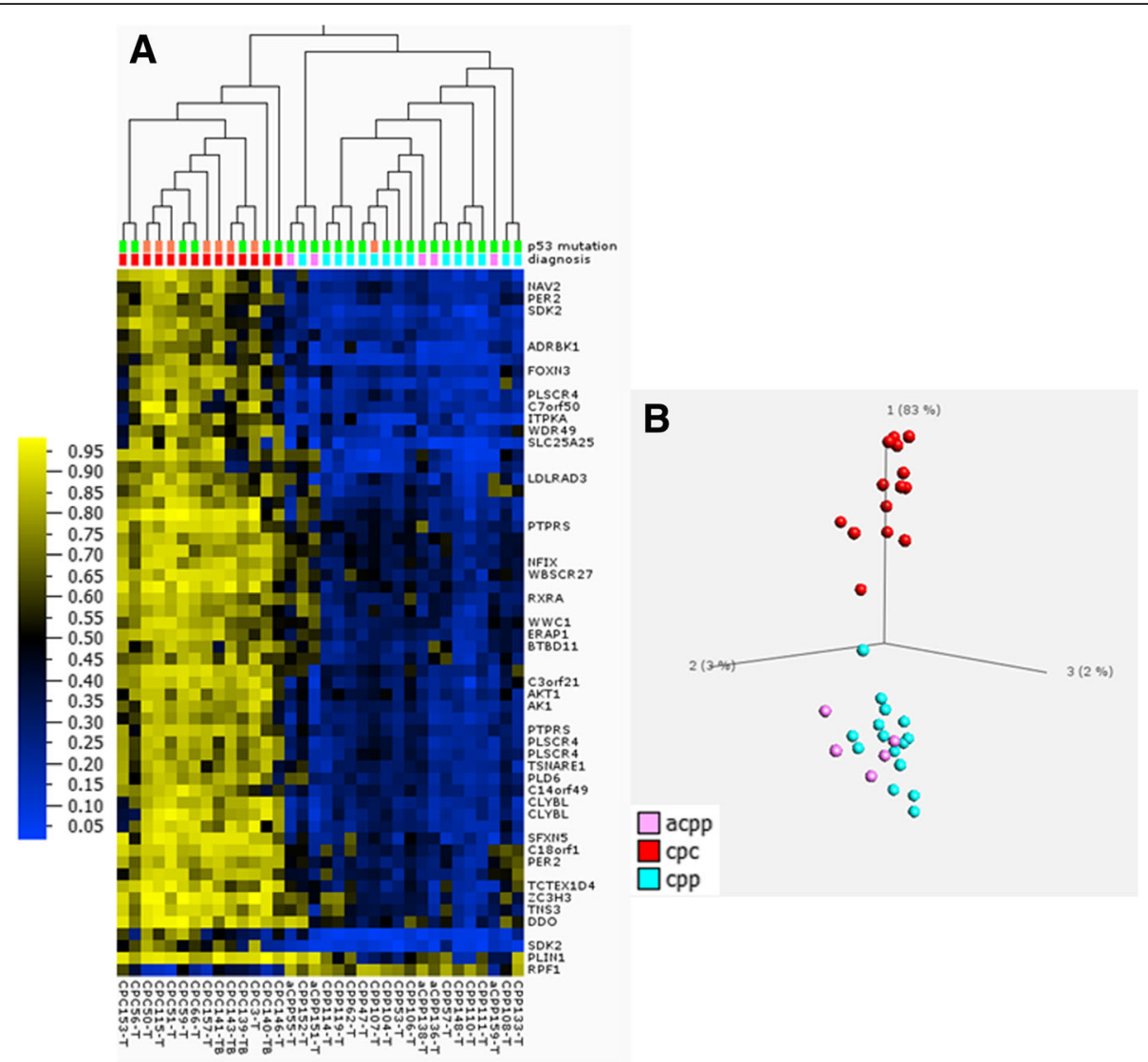

Fig. 5 CPC specific DNA methylation signature of 34 CPT samples Heatmap (a) and PCA (b) of 59 differentially methylated CpG sites encompassing 33 candidate genes extracted from the dataset of $3361 \mathrm{CpG}$ sites by applying increased stringency $(p<0.001$ and at least 0.4 delta beta). Hierarchical clustering was done using Euclidean metric. High methylation = yellow; low methylation = blue; TP53 status: wild type = green, mutated = orange; diagnosis: $\mathrm{cpc}=$ red, acpp = pink, $\mathrm{cpp}=$ turquoise. The numbers 1, 2, and 3 in PCA plot represent component 1, component 2, and component 3.

separate from CPPs. This indicates high sensitivity for the CPC-specific DNAm signature in properly assigning the histopathological profile to CPTs (Fig. 7a). Only two CPPs (CPP80g and CPP36g) clustered with the CPC; interestingly, the histopathological report for CPP36g showed that this specific CPP has increased cellularity, blurring of the papillary growth pattern, but no mitotic activity, no tumor necrosis, and no nuclear pleomorphism, features not commonly associated with CPP histopathology. This data suggests that the CPC-specific DNAm signature can be more specific in classifying subgroups of CPT tumors based on their methylation profiles compared to histopathological classification.

Furthermore, we wanted to check the sensitivity of our CPC-specific DNAm signature in classifying aCPPs from the replication cohort. The first methylation cluster includes most of the CPPs (cluster 1) and some aCPPs as expected. The second cluster is divided into two subclusters (cluster $2 \mathrm{~A}$ and $2 \mathrm{~B}$ ) where one is enriched in mostly CPCs (2B) and the other one (2A) includes a histologically defined mix of CPCs, CPPs, and aCPPs (Fig. 7b).
Hierarchical clustering performed separately for each histologically defined group of CPPs (Additional file 2: Figure S6A) and aCPPs (Additional file 2: Figure S6B) from the replication cohort is able to clearly distinguish a subset within each of the CPP and aCPP groups based on their DNAm profiles, although histologically they were all classified as either CPP or aCPP. Next, we combined samples from two cohorts (34 samples initially used in the discovery of the CPC specific DNAm signature and 61 samples from the replication cohort) to generate a DNAm profile based on the CPC-specific signature (Fig. 8a). Using heatmap combined with hierarchical clustering, the data shows that CPT samples are grouped into two main clusters (cluster1 and 2) but cluster 2 is divided into two subgroups (2A and $2 \mathrm{~B})$. The distribution of histologically defined CPCs, CPPs, and aCPPs is similar as described above when only a replication cohort of 61 samples was used (Fig. 7b). Based on the distribution of 95 histologically defined CPC, $\mathrm{CPP}$, and aCPP samples within molecularly defined clusters, we also looked at the frequency of either death or recurrence events. The frequency of a death event (Fig. 8b) is 

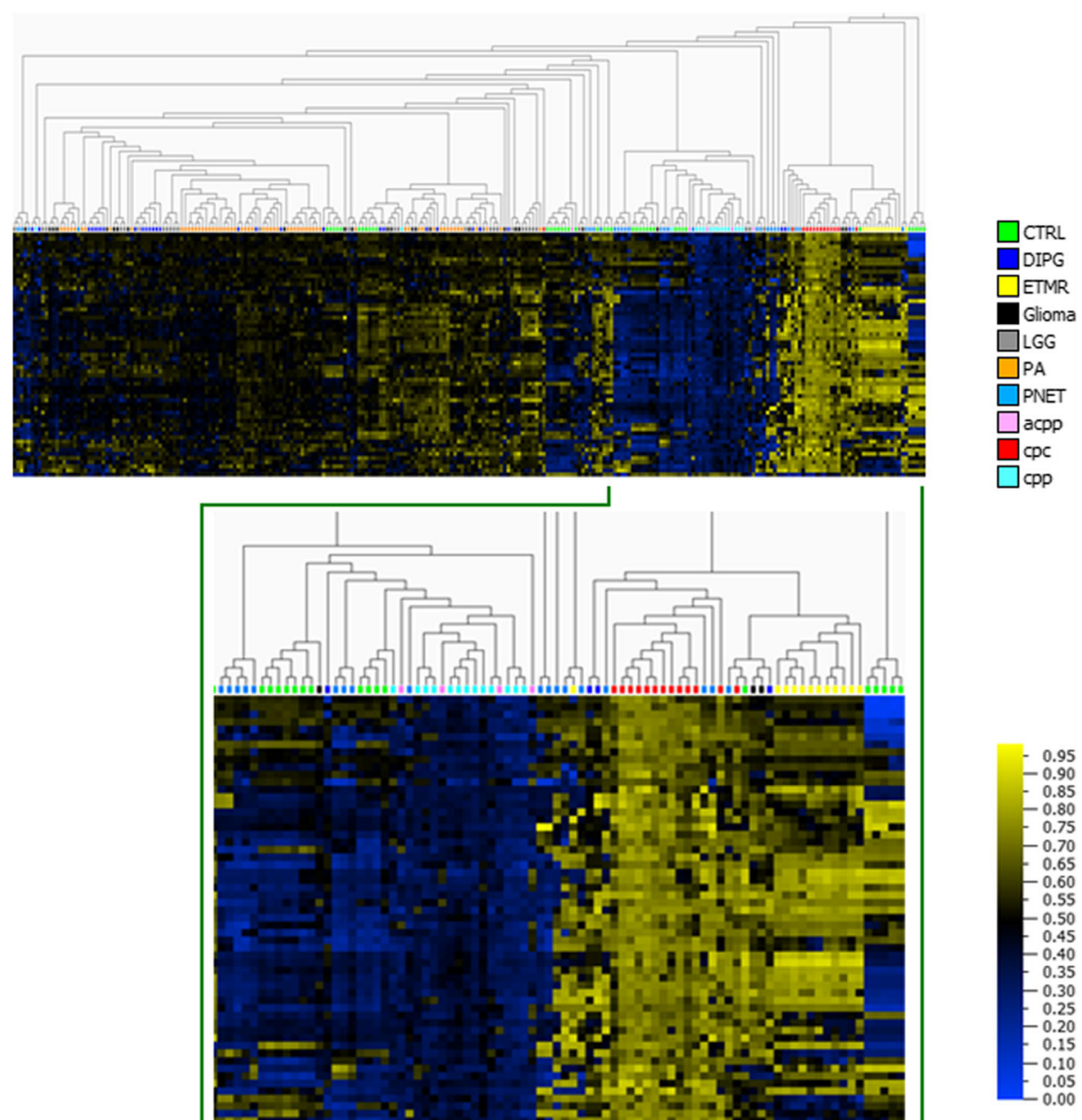

Fig. 6 Heatmap of brain tumor DNA methylation datasets from GEO and TCGA databases with CPC-specific DNA methylation signature (59 differentially methylated CPG sites). Comparison of CPC-specific DNA methylation signature to other brain tumors. Brain tumor data were derived from GEO database under accession number GSE50022—for diffuse intrinsic pontine glioma (DIPG) ( $n=28)$, GSE44684-from pilocytic astrocytoma (PA) $n=61$-PA and $n=6$ normal cerebellum (CTRL)), and GSE52556-from embryonal tumors with multilayered rosettes (ETMR) $(n=12)$, primitive neuroectodermal tumors (PNET) $(n=28)$, normal brain (CTRL) $(n=34)$, and TCGA-glioma ( $n=24)$ and low grade glioma (LGG) $(n=30)$ from TCGA. CPTs diagnosis: $c p c=$ red, acpp = pink, $c p p=$ turquoise. Hierarchical clustering was done using Euclidean metric. High methylation = yellow; low methylation = blue

very different in each of the molecularly defined groups. In group 1 , the frequency of a death event is $0 \%$, whereas it is $30 \%$ in group $2 \mathrm{~B}$ and only $7 \%$ in group $2 \mathrm{~A}$. The frequency of recurrence is $5 \%$ in group 1 , (Fig. 8 c) $61 \%$ in group $2 \mathrm{~B}$, and only $17 \%$ in group $2 \mathrm{~A}$. Frequency of either event is extremely high in group $2 \mathrm{~B}$, extremely low in group 1 , and significantly reduced in group 2A. Chances of surviving in group $2 \mathrm{~A}$ are 4 times higher than in group $2 \mathrm{~B}$. When estimating either death or recurrence events assessed on histologically defined CPTs, the death event was $28.1 \%$ in CPCs and $0 \%$ in CPPs or aCPPs (Fig. 8d) whereas the frequency of recurrence was $12.9 \%$ lower than projected based on DNAm clusters (Fig. 8e). DNA methylationbased disease risk assessment was significantly improved compared to the frequency of death event assessed on histologically defined CPTs. Seven percent $(\mathrm{CPP}=3$, $\mathrm{aCPP}=8, \mathrm{CPC}=5$ ) of patients could be classified with more favorable outcome compared to $0 \%$ when using histopathological criteria alone (Fig. 8d), while the recurrence frequency measured by current criteria was underestimated (Fig. 8e) when compared to frequency assessment based on specific DNAm signature for CPCs. Interestingly, factor analysis revealed that the CPC-specific DNAm signature forms an independent group of data, quite distinct from the available phenotype, genotype, or clinical attributes (Additional file 2: Figures S7 and S8). See Additional file 3 for details. We tested the methylation profiling classifier [12] (Heidelberg classifier at https://www.molecularneuropathology.org) on all samples from cluster 1, 2A, and $2 \mathrm{~B}$ in Fig. 8 to compare to the CPC-specific DNAm 


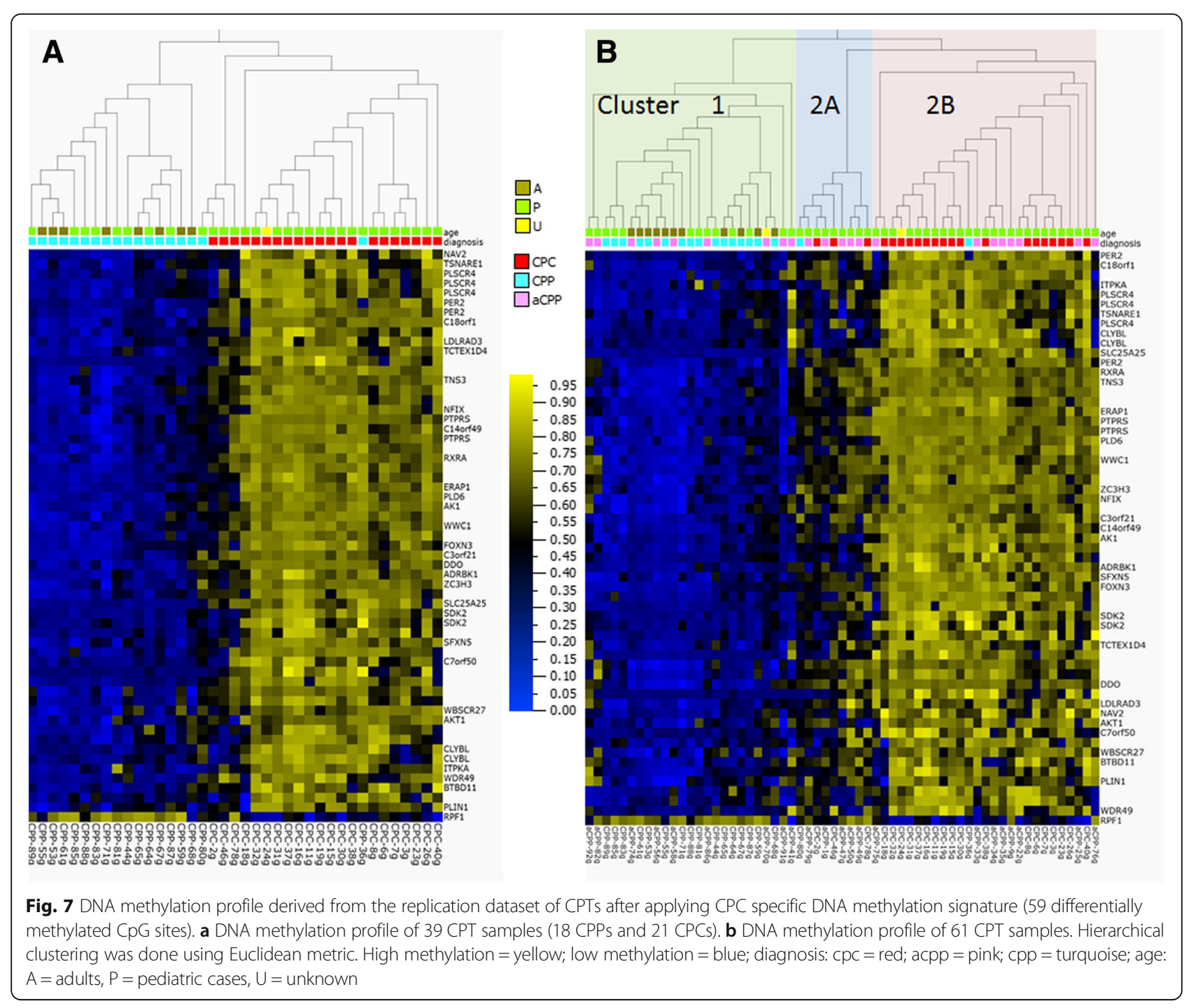

signature classification. The results are presented in Additional file 1: Table S9. In our cluster 2A, we found 5 CPCs which were classified by the Heidelberg classifier [12] as pediatric B (methylation class closely related to methylation cluster 3 described in Thomas et al. [13]). These CPCs represent a group of patients with much more favorable outcome then predicted by the Heidelberg classifier. We also found 3 CPPs and 2 aCPPs from our cluster 1 being classified as pediatric B. Our classification performed better than the Heidelberg classification given that a subset of the $\mathrm{CPCs} / \mathrm{CPP} / \mathrm{aCPPs}$ with favorable outcome was mixed among the ones with the severe outcome.

\section{Discussion}

We generated a genome-wide DNAm profile in CPTs to address our hypothesis that epigenetic alterations would be associated with specific phenotypes. We undertook a detailed approach to define the differences in DNAm signature for different groups of CPTs as one of the epigenetic factors responsible for CPC development and progression.

We showed significant genome-wide hypermethylation in CPCs compared to either CPPs or aCPPs, but no difference between CPPs and aCPPs. This could be in part due to the fact that we had a smaller number of aCPP cases. A total of 3361 differentially methylated probes showed segregation between the majority of CPCs and CPPs or aCPPs, thus distinguishing the aggressive form of CPTs, that is, CPCs from benign CPTs (CPPs or aCPPs). Unsupervised hierarchical clustering revealed two main clusters within CPCs based on TP53 mutation status. CPCs with homozygous TP53 mutations segregated from CPCs with heterozygous TP53 mutations or CPCs with TP53-wt and showed significantly worse survival outcome compared to CPPs and aCPPs or CPCs with heterozygous TP53 mutations or TP53-wt. This is consistent with our previous observation of the worst 


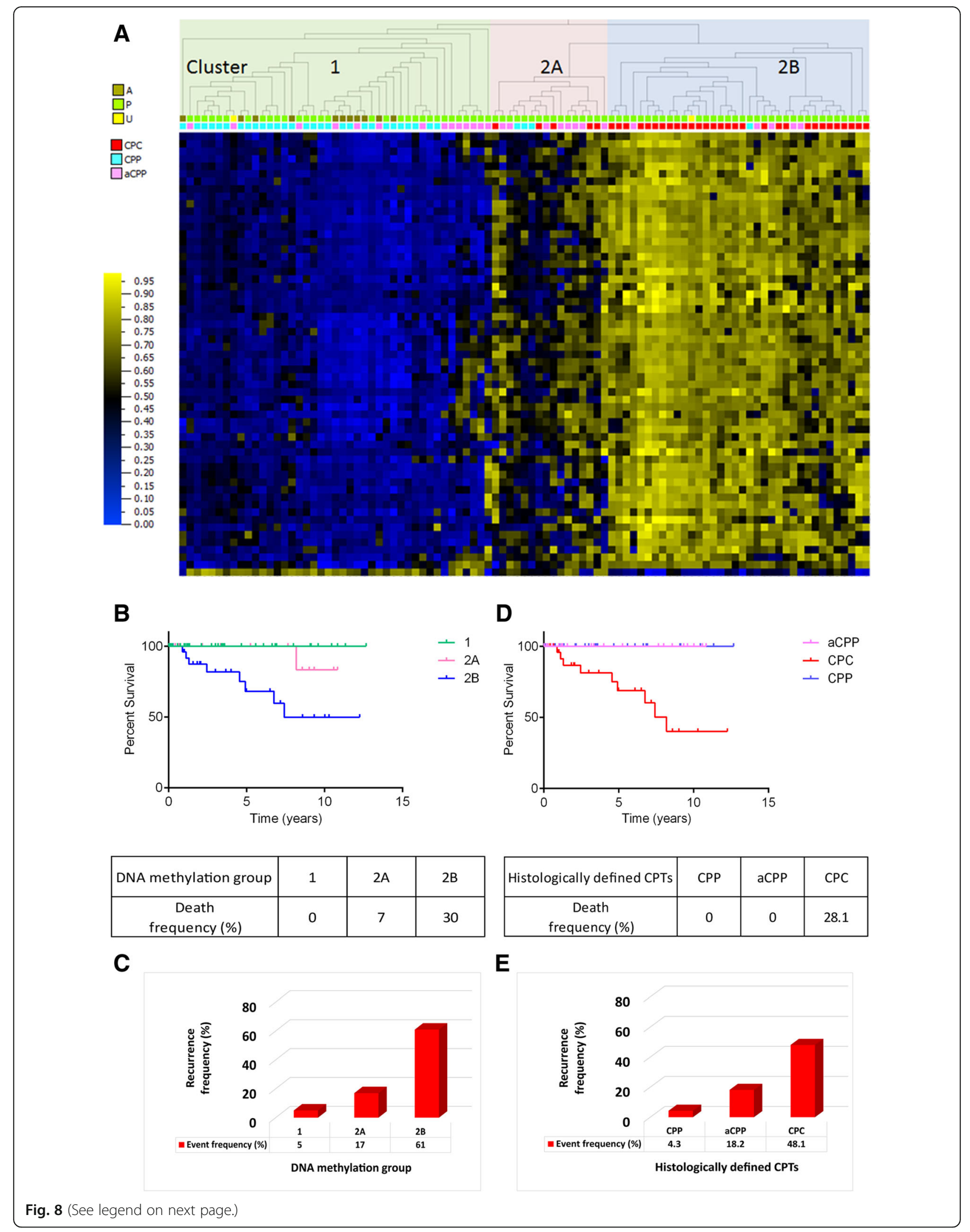


(See figure on previous page.)

Fig. 8 DNA methylation profile derived from the combined discovery and replication datasets of CPTs after applying CPC specific DNA methylation signature (59 differentially methylated CpG sites), Kaplan-Meier plots showing overall survival by methylation subgroups or by histological diagnosis along with tables showing frequency of death event and histograms showing frequency of recurrence events within each of the DNA methylation signature derived clusters as well as for each of the histologically defined CPTs. a DNA methylation clusters of the 95 CPT samples (combined datasets of 34 samples used in discovery cohort and 61 samples from replication cohort) defined by applying CPC-specific DNA methylation signature. Hierarchical clustering was done using Euclidean metric. High methylation = yellow; low methylation = blue; diagnosis: $c p c=r e d$, acpp $=p i n k$, $c p p=t$ turquoise; age: $A$ = adults, $P$ = pediatric cases, $U$ = unknown. Kaplan-Meier curves depicting overall survival (OS) estimates of patients with CPT by methylation subgroups (b) or by histological diagnosis $(\mathbf{d})$. Statistical values were obtained with the log-rank chi-square $=11.8(\mathrm{df}=1), p$ value 0.0008 , when comparing patients grouped by methylation clusters and with the log-rank chi-square $=0.003495(\mathrm{df}=2), p$ value 0.9529 , when comparing patients grouped by diagnosis. Histograms showing frequency of recurrence event in each of the clusters derived from DNA methylation signature (c) of 95 CPT samples or for each of the histologically defined CPTs (e)

outcome for CPC tumors carrying homozygous TP53 mutations [9]. Some TP53-wt CPCs cluster within group 2 (together with heterozygous mutated TP53) and in our cohort one of the TP53-wt patients did not survive, but two TP53-wt CPC patients cluster out of the CPC clusters (1 or 2), with CPPs in cluster 3 showing excellent survival. It is hard to predict, based on TP53 mutational status alone, which CPC patient with TP53-wt would have a better prognosis. By combining DNAm and TP53 mutational status, we show that we can distinguish between TP53-wt cases and predict the outcome.

Pathway analysis using IPA identified nine canonical pathways with GABA receptor at the top of the list that were significantly enriched in CPCs in comparison with CPPs or aCPPs. GABA neurotransmitter in the mammalian central nervous system (CNS) acts at either ionotropic (GABA(A)Rs) or metabotropic (GABA(B)Rs) receptors.

Intriguingly, GABA has also emerged as a tumor signaling molecule in the brain and periphery that controls tumor cell proliferation $[17,18]$. Recent studies indicate that GABA and its receptors seem to play regulatory effects in many kinds of cancers [19, 20]. Observations that $\mathrm{GABA}(\mathrm{A})$ receptor levels change in different tumors and tumor cell lines raise the possibility that manipulating GABA receptor activity might inhibit tumor growth [21]. The GABA(A) receptor allosteric agonist Nembutal has been shown experimentally to inhibit colon cancer growth and metastasis [22]. GABA also plays a role in synchronizing suprachiasmatic nucleus (SCN) neurons [23], and recent evidence suggesting the presence of rhythmic clock gene expression in rat choroid plexus (CP) points to the possible involvement of $\mathrm{CP}$ in SCN circadian information [24]. It is possible that deregulated GABR(A) signaling in CPTs has an effect on the choroidal autonomous clock or on synchronization signals/ circuits from the SCN neurons. Since the circuits are critical for regulating physiology and behavior, as well as the integration of metabolic information [25], disturbances in the communication between the body clocks can desynchronize the circadian system, which is believed to contribute to the development of many diseases including cancer [26].
In this study, we demonstrate that DNAm profiling can distinguish aggressive forms of CPTs from benign forms of CPTs. We have generated a CPC-specific DNAm signature which includes several important genes such as $A K 1$, PER2, and PLSCR4. This signature is highly specific when compared to other brain tumors highlighting its potential clinical diagnostic utility. When the CPC-specific signature was applied to a replication cohort of 39 samples (18 CPPs and 21 CPCs), CPCs were clustered together, separate from CPPs, but 2 histologically defined CPPs (CPP80g and CPP36g) clustered with CPCs. Thus, we are able to distinguish two distinct molecular subgroups, one comprised of a mixed population of histopathologically defined samples with a majority of one histologically defined subtype and one homogenous subgroup entirely comprised of CPPs.

Also, checking sensitivity of the CPC-specific DNAm signature in classifying the aCPP in the replication cohort of 61 samples (18 CPPs, $21 \mathrm{CPCs}$, and 22aCPPs), we found that aCPPs are distributed across different molecular subgroups. These data strongly suggest that our CPCspecific DNAm signature is more accurate in classifying CPT tumors based on their methylation profiles compared to histopathological classification alone and that the WHO classification of the CPT based on CPP, aCPP, and $\mathrm{CPC}$ can benefit from the addition of the molecular signature to provide a more accurate diagnosis that could impact patient clinical management.

The CPC-specific DNAm signature we described here comprised several genes including $A K 1, P E R 2$, and PLSCR4. Methylation of the CpG sites in the promoter regions of these genes was more than $30 \%$ higher in CPC than in CPPs or aCPPs. This was correlated with significant downregulation at the mRNA level indicating that the transcription of these genes is epigenetically regulated in CPCs.

Emerging evidence has also revealed tight links between the regulation of cellular metabolism [27] and the molecular clock and that the alteration of circadian rhythms might lead to increased susceptibility to cancer in humans $[26,28]$. AK1 deficiency, which we observed in our previous study [9], is known to reduce metabolic signal 
reception by metabolic sensors, such as K-ATP channels and AMP-activated protein kinase (AMPK), compromising their ability to withstand energetic stress $[29,30]$. In the context of a circadian clock, AMPK is essential for maintaining metabolic homeostasis and preventing metabolic disorders [31].

The mechanism of dysregulation of circadian genes in cancers includes epigenetic silencing by promoter methylation. We suggest that loss of circadian homeostasis through circadian disruption may be a novel risk factor in choroid plexus tumorigenesis. Decreased expression through hypermethylation of $\mathrm{CpG}$ islands or aberrant acetylation in the promoters of the core circadian genes Per1, Per2, and Per3 are reported in a spectrum of human cancers [32]. More studies are needed to test the hypothesis that loss of circadian homeostasis through circadian disruption may be a potential risk factor in choroid plexus tumorigenesis.

In our study, hypermethylation of CpG sites in the promoter region of $P E R 2$ is correlated with significantly lower expression of PER2 in CPCs compared to CPPs or aCPPs. PER1 and Cryptochrome-2 (CRY2) genes belonging to the core clock family of genes showed significantly lower expression in CPCs as well [9].

PER1 and PER2 genes are currently considered to be true tumor suppressor genes, as decreased expression of either (or both) has been reported in several types of human cancers [33-35] and has been shown to be an independent predictor of poor prognosis in patients with gastric cancer [36].

Downregulation of Per2 is correlated with increased levels of Cyclin D and Cyclin E and accelerated tumor growth in vivo [37] whereas induced overexpression of either Per1 or Per2 has been shown to inhibit the growth of cancer cells and increase their apoptotic rate [38]. In our previous study [9], we observed increased levels of Cyclin $A 2$ as well as Cyclin E1 and E2 in CPCs compared to CPPs or aCPPs further confirming a correlation between downregulation of $P E R 2$ and increased levels of Cyclin $A$ and Cyclin $E$ as one of the factors involved in CPCs tumorigenesis.

The findings generated from this study provide a framework for improved molecular stratification for diagnosis and treatment as well as the development of potential prognostic markers to better differentiate aggressive CPT tumors from those that are not life threatening. As well, some of the markers might be predictors of response to particular chemotherapeutic agents as previously reported [39].

Furthermore, our study confirms the relationship between the circadian clock, cancer, and DNAm at clock genes and suggests that improper DNAm may alter clock gene expression, contributing to the etiology of CPCs. The distinct proliferation rhythm between tumor cells and normal cells [40] provides an intriguing opportunity to maximize the effect of anticancer therapies in CPC based on this circadian clock.

In conclusion, we discovered a highly sensitive and specific DNAm signature for CPCs which is able to segregate CPC not only from other CPTs but also from other brain tumors. However, the study is not able to differentiate the proportion of the non-tumor cells. We demonstrate that heterogeneity in the clinical outcome of CPT patients cannot be predicted by histopathological assessment alone, but implementation of the specific DNAm signature for CPCs in association with the morphology of the CPT tumor can significantly improve diagnosis. This signature needs to be validated with larger sample numbers before being used in the clinical setting; however, it shows its usefulness in identifying cases which would otherwise be undertreated or perhaps some which would be overtreated. The intention of making this signature accessible to the scientific-medical community raises awareness of the existence of this potentially more accurate classification.

Incorporation of CPC-specific DNAm signature into existing survival prediction based solely on histopathological criteria can significantly improve the estimation of disease outcome. Seven percent of patients could be classified as lower risk compared to $0 \%$ when using histopathological criteria alone, while the recurrence frequency measured by current criteria was underestimated when compared to frequency assessment based on specific DNAm signature for CPCs.

DNA methylation profiling enables the subclassification of CPTs into 3 disease subgroups in routinely collected material, and the integration of CPC-specific DNAm signature can significantly improve prognostic risk prediction allowing for informed treatment decision, protecting some young patients from devastating and permanent neurological impairment due to aggressive treatment.

\section{Additional files}

Additional file 1: Table S1. Patient information for samples used in DNA methylation experiment. Table S2. Patient information for samples in replication cohort used in sensitivity testing of the CPC DNA methylation signature. Table S3. Multivariate factor analysis. Table S4. Primer sets for quantitative sodium bisulfite pyrosequencing. Table S5. GABA receptor DNA methylation status in CPTs. Table S6. Differentially methylated genes in cpc vs cpp showing differential expression and reverse correlation with DNA methylation in the promoter region of the gene. Table S7. Differentially methylated genes in cpc vs cpp showing differential expression and positive correlation with DNA methylation in the body of the gene. Table S8. 39 variables (encompassing 33 single genes) segregating CPCS and CPPs. Table S9. Comparison between CPC-specific DNAm signature classification and the Heidelberg classifier. (XLSX $108 \mathrm{~kb}$ )

Additional file 2: Figure S1. Volcano plot showing significantly differentially methylated CpGs (yellow and blue) between CPCs (carcinomas) and CPPs (papilomas). The $X$-axis shows the difference between average DNAm levels in carcinomas and in papilomas, whereas the $Y$-axis shows the significance as the Mann-Whitney $U p$ value (on the 
logarithmic scale). Each point represents a CpG position from the Illumina HumanMethylation450 BeadChip. CPC tumors show an overall predominance of hypermethylation (yellow) compared with CPPs across the signature $\mathrm{CpGs}$, which were identified using the significance level $p<0.05$ (or $\log 10(p)>1.30$ ) and DNA methylation difference of at least $30 \%$. Figure S2. Functional genomic distribution of CpG sites in CPCs. Genomic enrichment of the CPC DNA methylation signature is presented as percentage of all $\mathrm{CpG}$ sites on HumanMethylation450 BeadChip from Illumina (green) or of $\mathrm{CpG}$ sites derived from differential analysis using corrected Mann-Whitney U $p$ value $<0.05$ and at least $30 \%$ difference in average beta between CPCS and CPPs (orange). Figure S3. BoxPlot showing differential methylation of tested $\mathrm{CpG}$ sites between $\mathrm{CPCS}$ and CPPs or aCPPs. (A) Ak1-cg14578146, (B) PER2-cg11903188, (C) PLSCR4cg07038342; the number of samples in discovery set was 34 and in validation set 22; $Y$-axis shows average beta values and $X$-axis tumor type: $C P C=$ red, $a C P P=$ pink, and $C P P=$ turquoise. $p$ value was generated using two group comparison (t test) and represents significance

of the difference in methylation between CPCS and CPPs or aCPPs. ${ }^{* * *} p<0.001$. Figure $\mathbf{S 4}$. CPC-specific minimal DNA methylation signature. Heatmap (A) and PCA (B and C) of 3 differentially methylated $\mathrm{CpG}$ sites encompassing 3 candidate genes from the dataset of $59 \mathrm{CpG}$ sites of CPC-specific signature. This minimal signature shows segregation between CPCs and CPPs or aCPPs. Hierarchical clustering was done using Euclidean metric. High methylation = yellow, low methylation = blue, discovery set (lllumina HumanMethylation450 BeadChip) on 34 discovery samples $=$ orange, validation set (targeted quantitative sodium bisulfite pyrosequencing) on 34 discovery, and 22 validation samples = green; diagnosis: $\mathrm{cpc}=$ red, acpp = pink, and $\mathrm{cpp}=$ turquoise. The numbers 1, 2 , and 3 in PCA plots

represent component 1, component 2, and component 3. Figure S5. Correlation plot of DNA methylation values (\%) in 34 DNA samples for each tested CpG obtained using the Illumina HumanMethylation450 BeadChip and pyrosequencing. High correlation between the two methodologies was observed with an $r^{2}$ value of $\geq 0.9 ; r^{2}$-Pearson's correlation coefficient.

Figure S6. Hierarchical clustering of DNA methylation profile performed separately for each histologically defined group of 18 CPPs (6A) and 22 aCPPs (6B) from replication cohort. Hierarchical clustering was done using Euclidean metric. High methylation =yellow, low methylation = blue; diagnosis: $\mathrm{cpp}=$ turquoise and $\mathrm{acpp}=$ pink. Figure S7. Factor analysis of DNAm signature along with phenotype and genotype sample attributes. The heatmap shows the magnitude of factor loadings for $n=12$ factors in each of the data attribute. The DNAm beta values at 59 CpG sites contribute strongly to factors $1-3$ and $6-12$, for which no other available data attributes contribute. Factor 4 is associated with recurrence status, death status, and P53 mutation status, and factor 5 with age. Several $\mathrm{CpGs}$ also show an association with these phenotype attributes, but generally less so that with the DNAmrelated factors 1-3. Figure S8. Factor analysis of DNAm signature along with phenotype and genotype sample attributes, with recurrence attribute removed. The heatmap shows the magnitude of factor loadings for $n=12$ factors in each of the data attribute. Factor 4 is associated with age, and factor 6 with death status and P53 mutation status. The DNAm beta values at $59 \mathrm{CpG}$ sites contribute strongly to the rest of the factors, with the strongest association being in the top three factors. (DOCX $2180 \mathrm{~kb}$ )

Additional file 3: Multivariate factor analysis. (DOCX $16 \mathrm{~kb}$ )

\section{Abbreviations}

aCPP: Atypical choroid plexus papilloma; AK1: Adenylate kinase; AMPK: AMP-activated protein kinase; CDMR: Cancer-specific differentially methylated regions; CP: Choroid plexus; CPC: Choroid plexus carcinoma; CpG: Cytosinephosphate-guanine; CPP: Choroid plexus papilloma; CPTs: Choroid plexus tumors; CRY2: Cryptochrome-2; DIPG: Intrinsic pontine gliomas; DNA: Deoxyribonucleic acid; DNAm: DNA methylation; FFPE: Formalin-fixed paraffin-embedded; GEO: Gene Expression Omnibus; IPA: Ingenuity Pathway Analysis; LFS: Li-Fraumeni syndrome; LGG: Low grade glioma; LOO: Leaveone-out; OCT: Optimal cutting temperature; OS: Overall survival; PCA: Principal component analysis; PER2: Period circadian clock 2; PLSCR4: Phospholipid scramblase; PNET: Primitive neuroectodermal tumors: rDMR: Reprogrammed-specific differentially methylated regions;
TCAG: Centre for Applied Genomics; TCGA: The Cancer Genome Atlas; TP53wt: Wild type TP53; TSS: Transcription start site; WHO: World Health Organization

\section{Authors' contributions}

MP, SC, and DM conceived and designed the study and prepared the manuscript. MP, SC, ALT, TG, and DMM performed DNA methylation studies and statistical and bioinformatics analysis. AN, AS, CH, EB, UT, RG, NJ, DM, CT, $M S, D C$, and $M H$ provided the clinical data. RG, JLF, NJ, MB, CT, MS, DC, MH, RW, and DM provided the critical review of the manuscript. All authors have read and commented on the manuscript and approved the final version.

\section{Funding}

This research is supported by a grant from the Canadian Institute for Health Research (CIHR): 6210100362. Bioinformatics analyses were supported in part by the Canadian Centre for Computational Genomics (C3G), part of the Genome Technology Platform (GTP), funded by Genome Canada through Genome Quebec and Ontario Genomics (ALT and MB).

\section{Availability of data and materials}

Genome-wide DNAm profiles for all 34 primary samples are available through Gene Expression Omnibus (GEO: http://www.ncbi.nlm.nih.gov/geo/), accession number GSE61044.

\section{Ethics approval and consent to participate}

Ethics approval was obtained for the study from the Institutional Research Ethics Board. Informed consent was obtained from the parents/legal guardians of all patients.

\section{Consent for publication}

Not applicable.

\section{Competing interests}

The authors declare that they have no competing interests.

\section{Author details}

${ }^{1}$ Genetics and Genome Biology Program, Hospital for Sick Children, PGCRL, 686 Bay Street, Toronto, Ontario M5G 0A4, Canada. ${ }^{2}$ Center for Computational Medicine, Hospital for Sick Children, PGCRL, 686 Bay Street, Toronto, Ontario M5G 0A4, Canada. ${ }^{3}$ Friends of Cancer Research, $1800 \mathrm{M}$ Street, NW, Suite 1050 South, Washington, DC 20036, USA. ${ }^{4}$ Department of Computer Science, University of Toronto, 40 St. George Street, Toronto, Ontario M5S 2E4, Canada. ${ }^{5}$ Division of Clinical and Metabolic Genetics, Hospital for Sick Children, 555 University Avenue, Toronto, Ontario M5G 1X8, Canada. ${ }^{6}$ Paediatric Laboratory Medicine, Hospital for Sick Children, 555 University Avenue, Toronto, Ontario M5G 1X8, Canada. ${ }^{7}$ Division of Hematology/Oncology, Hospital for Sick Children, 555 University Avenue, Toronto, Ontario M5G 1X8, Canada. ${ }^{8}$ Department of Pediatrics, Hospital for Sick Children, 555 University Avenue, Toronto, Ontario M5G 1X8, Canada. ${ }^{9}$ Department of Medical Biophysics, University of Toronto, Princess Margaret Cancer Research Tower, MaRS Centre, 101 College Street, Toronto, Ontario M5G 1 L7, Canada. ${ }^{10}$ Department of Oncology, Cambridge Cancer Center, Robinson Way, Cambridge CB2 ORE, England. " ${ }^{11}$ Neuro-Oncology Program, Nationwide Children's Hospital and The Ohio State University, 700 Children's Dr, Columbus, OH 43205, USA. ${ }^{12}$ Division of Hematology/Oncology, Montreal Children's Hospital of the McGill University Health Centre (RI-MUHC), 1001 Decarie Blvd, Montreal, Québec H4A 3 J1, Canada. ${ }^{13}$ Institute of Neuropathology, University Hospital Münster, 48149 Münster, Germany. ${ }^{14}$ Hopp Children's Cancer Center at the NCT Heidelberg (KiTZ), Im Neuenheimer Feld 280, 69120 Heidelberg, Germany. ${ }^{15}$ Division of Pediatric Neurooncology, German Cancer Research Center (DKFZ) and German Cancer Consortium (DKTK), Im Neuenheimer Feld 280, 69120 Heidelberg, Germany. ${ }^{16}$ Charité Universitätsmedizin Berlin, corporate member of Freie Universität Berlin, Humboldt-Universität zu Berlin, and Berlin Institute of Health, Department of Neuropathology, Charitéplatz 1, 10117 Berlin, Germany. ${ }^{17}$ German Cancer Consortium (DKTK), Partner Site Berlin, Invalidenstrasse 80, 10117, Berlin, German Cancer Research Center (DKFZ), Im Neuenheimer Feld 280, 69120 Heidelberg, Germany. 
Received: 30 January 2019 Accepted: 22 July 2019

10.

\section{References}

1. Louis DN, Ohgaki H, Wiestler OD, Cavenee WK, Burger PC, Jouvet A, et al. The 2007 WHO classification of tumours of the central nervous system. Acta Neuropathol. 2007:114(2):97-109.

2. Rickert $\mathrm{CH}$, Paulus W. Tumors of the choroid plexus. Microsc Res Tech. 2001;52(1):104-11.

3. Berger C, Thiesse P, Lellouch-Tubiana A, Kalifa C, Pierre-Kahn A, Bouffet E. Choroid plexus carcinomas in childhood: clinical features and prognostic factors. Neurosurgery. 1998;42(3):470-5.

4. Fitzpatrick LK, Aronson $L$. Cohen $\mathrm{KJ}$. Is there a requirement for adjuvant therapy for choroid plexus carcinoma that has been completely resected? J Neurooncol. 2002;57(2):123-6.

5. Wrede B, Liu P, Wolff JE. Chemotherapy improves the survival of patients with choroid plexus carcinoma: a meta-analysis of individual cases with choroid plexus tumors. J Neurooncol. 2007;85(3):345-51.

6. Wolff JE, Sajedi M, Coppes MJ, Anderson RA, Egeler RM. Radiation therapy and survival in choroid plexus carcinoma. Lancet. 1999; 353(9170):2126

7. Tabori U, Shlien A, Baskin B, Levitt S, Ray P, Alon N, et al. TP53 alterations determine clinical subgroups and survival of patients with choroid plexus tumors. J Clin Oncol. 2010;28(12):1995-2001.

8. Bougeard G, Renaux-Petel M, Flaman JM, Charbonnier C, Fermey P, Belotti $\mathrm{M}$, et al. Revisiting Li-Fraumeni syndrome from TP53 mutation carriers. J Clin Oncol. 2015;33(21):2345-52.

9. Merino DM, Shlien A, Villani A, Pienkowska M, Mack S, Ramaswamy V, et al. Molecular characterization of choroid plexus tumors reveals novel clinically relevant subgroups. Clin Cancer Res. 2015;21(1):184-92.

10. Schwalbe EC, Lindsey JC, Nakjang S, Crosier S, Smith AJ, Hicks D, et al. Novel molecular subgroups for clinical classification and outcome prediction in childhood medulloblastoma: a cohort study. Lancet Oncol. 2017;18(7):958-71.

11. Choi W, Lee J, Lee JY, Lee SM, Kim DW, Kim YJ. Classification of colon cancer patients based on the methylation patterns of promoters. Genomics Inform. 2016;14(2):46-52.

12. Capper D, Jones DTW, Sill M, Hovestadt V, Schrimpf D, Sturm D, et al. DNA methylation-based classification of central nervous system tumours. Nature. 2018:555(7697):469-74.

13. Thomas C, Sill M, Ruland V, Witten A, Hartung S, Kordes U, et al. Methylation profiling of choroid plexus tumors reveals 3 clinically distinct subgroups. Neuro Oncol. 2016;18(6):790-6.

14. Chen YA, Lemire M, Choufani S, Butcher DT, Grafodatskaya D, Zanke BW, et al. Discovery of cross-reactive probes and polymorphic CpGs in the Illumina Infinium HumanMethylation450 microarray. Epigenetics. 2013;8(2):203-9.

15. Kuhn MA, Olexa T, Dhawan A. Building predictive models in R Using the caret package. Arthroscopy. 2008;24(11):1299-300.

16. Choufani S, Shapiro JS, Susiarjo M, Butcher DT, Grafodatskaya D, Lou $Y$, et al. A novel approach identifies new differentially methylated regions (DMRs) associated with imprinted genes. Genome Res. 2011; 21(3):465-76.

17. Szczaurska K, Mazurkiewicz M, Opolski A. The role of GABA-ergic system in carcinogenesis. Postepy Hig Med Dosw. 2003;57(5):485-500.

18. Watanabe M, Maemura K, Oki K, Shiraishi N, Shibayama Y, Katsu K. Gammaaminobutyric acid (GABA) and cell proliferation: focus on cancer cells. Histol Histopathol. 2006;21(10):1135-41.

19. Schuller HM, Al-Wadei HA, Majidi M. Gamma-aminobutyric acid, a potential tumor suppressor for small airway-derived lung adenocarcinoma. Carcinogenesis. 2008;29(10):1979-85.

20. Abdul M, McCray SD, Hoosein NM. Expression of gamma-aminobutyric acid receptor (subtype A) in prostate cancer. Acta Oncol. 2008;47(8): 1546-50.

21. Young SZ, Bordey A. GABA's control of stem and cancer cell proliferation in adult neural and peripheral niches. Physiology (Bethesda). 2009:24:171-85.

22. Thaker PH, Yokoi K, Jennings NB, Li Y, Rebhun RB, Rousseau DL Jr, et al. Inhibition of experimental colon cancer metastasis by the GABA-receptor agonist nembutal. Cancer Biol Ther. 2005;4(7):753-8.
23. Evans JA, Leise TL, Castanon-Cervantes O, Davidson AJ. Dynamic interactions mediated by nonredundant signaling mechanisms couple circadian clock neurons. Neuron. 2013;80(4):973-83.

24. Quintela T, Sousa C, Patriarca FM, Goncalves I, Santos CR. Gender associated circadian oscillations of the clock genes in rat choroid plexus. Brain Struct Funct. 2015;220(3):1251-62.

25. Albrecht U. Timing to perfection: the biology of central and peripheral circadian clocks. Neuron. 2012;74(2):246-60.

26. Stevens RG. Circadian disruption and breast cancer: from melatonin to clock genes. Epidemiology. 2005;16(2):254-8.

27. Green CB, Takahashi JS, Bass J. The meter of metabolism. Cell. 2008; 134(5):728-42.

28. Hansen J. Increased breast cancer risk among women who work predominantly at night. Epidemiology. 2001;12(1):74-7.

29. Carrasco AJ, Dzeja PP, Alekseev AE, Pucar D, Zingman LV, Abraham MR, et al. Adenylate kinase phosphotransfer communicates cellular energetic signals to ATP-sensitive potassium channels. Proc Natl Acad Sci U S A. 2001: 98(13):7623-8

30. Hancock CR, Janssen E, Terjung RL. Contraction-mediated phosphorylation of AMPK is lower in skeletal muscle of adenylate kinase-deficient mice. J Appl Physiol (1985). 2006;100(2):406-13.

31. Lee Y, Kim EK. AMP-activated protein kinase as a key molecular link between metabolism and clockwork. Exp Mol Med. 2013;45:e33.

32. Chen ST, Choo KB, Hou MF, Yeh KT, Kuo SJ, Chang JG. Deregulated expression of the PER1, PER2 and PER3 genes in breast cancers. Carcinogenesis. 2005;26(7):1241-6.

33. Hsu CM, Lin SF, Lu CT, Lin PM, Yang MY. Altered expression of circadian clock genes in head and neck squamous cell carcinoma. Tumour Biol. 2012; 33(1):149-55.

34. Mazzoccoli G, Piepoli A, Carella M, Panza A, Pazienza V, Benegiamo $G$, et al. Altered expression of the clock gene machinery in kidney cancer patients. Biomed Pharmacother. 2012;66(3):175-9.

35. Relles D, Sendecki J, Chipitsyna G, Hyslop T, Yeo CJ, Arafat HA. Circadian gene expression and clinicopathologic correlates in pancreatic cancer. J Gastrointest Surg. 2013:17(3):443-50

36. Zhao H, Zeng ZL, Yang J, Jin Y, Qiu MZ, Hu XY, et al. Prognostic relevance of period1 (Per1) and period2 (Per2) expression in human gastric cancer. Int J Clin Exp Pathol. 2014;7(2):619-30.

37. Yang X, Wood PA, Ansell CM, Quiton DF, Oh EY, Du-Quiton J, et al. The circadian clock gene Per1 suppresses cancer cell proliferation and tumor growth at specific times of day. Chronobiol Int. 2009;26(7): 1323-39.

38. Miyazaki K, Wakabayashi M, Hara Y, Ishida N. Tumor growth suppression in vivo by overexpression of the circadian component, PER2. Genes Cells. 2010;15(4):351-8.

39. Sandoval J, Esteller M. Cancer epigenomics: beyond genomics. Curr Opin Genet Dev. 2012;22(1):50-5.

40. You S, Wood PA, Xiong Y, Kobayashi M, Du-Quiton J, Hrushesky WJ. Daily coordination of cancer growth and circadian clock gene expression. Breast Cancer Res Treat. 2005;91(1):47-60.

\section{Publisher's Note}

Springer Nature remains neutral with regard to jurisdictional claims in published maps and institutional affiliations.

Ready to submit your research? Choose BMC and benefit from:

- fast, convenient online submission

- thorough peer review by experienced researchers in your field

- rapid publication on acceptance

- support for research data, including large and complex data types

- gold Open Access which fosters wider collaboration and increased citations

- maximum visibility for your research: over $100 \mathrm{M}$ website views per year

At $\mathrm{BMC}$, research is always in progress.

Learn more biomedcentral.com/submissions 